\title{
Association between baseline and changes in serum uric acid and incident metabolic syndrome: a nation-wide cohort study and updated meta-analysis
}

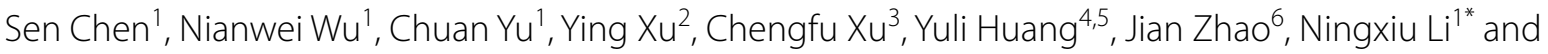
Xiong-Fei $\operatorname{Pan}^{5,7,8^{*}}$

\begin{abstract}
Background: To prospectively examine the associations of baseline serum uric acid (SUA) and SUA changes with incident metabolic syndrome (MetS) and update the evidence through a meta-analysis.

Methods: Our analyses were based on the China Health and Retirement Longitudinal Study from 2011-2012 to 2015-2016. The exposures were baseline SUA and SUA changes, and the outcome was incident MetS assessed in 2015-2016. Logistic regression was used to estimate odds ratios (ORs) and 95\% confidence intervals (Cls). A metaanalysis was conducted to synthesize evidence from all cohort studies on the same topic.

Results: Of 3779 participants (47.2\% men; mean age: 59.5 years) without MetS, 452 participants developed MetS after a follow-up of 4 years. Compared to the lowest quartiles, the adjusted ORs ( $95 \% \mathrm{Cls}$ ) for MetS were 1.08 (0.77-1.50), 1.32 (0.95-1.82), and 1.55 (1.12-2.16) for three higher quartiles of baseline SUA, and 1.23 (0.89-1.71), 1.39 (1.00-1.93), and 1.89 (1.38-2.58) for three higher quartiles of SUA changes. Each increment of $1 \mathrm{mg} / \mathrm{dL}$ of baseline SUA level was associated with 19\% higher odds of MetS (adjusted OR 1.19; 95\% Cl 1.07-1.33). In the meta-analysis of 24 cohort studies among 140,913 participants, the pooled relative risk (95\% Cl) was $1.32(1.25-1.40)$ for the highest versus lowest SUA category, and 1.15 (1.09-1.21) for each $1 \mathrm{mg} / \mathrm{dL}$ increase in the SUA level.

Conclusions: Both baseline SUA and longitudinal SUA changes were positively associated with risk of MetS among middle-aged and elderly Chinese, which was supported by findings from a comprehensive meta-analysis across multiple populations. SUA levels might need to be monitored closely for subsequent risk of MetS in clinical practice.
\end{abstract}

Keywords: Metabolic syndrome, Serum uric acid, Chinese, Cohort, Meta-analysis

*Correspondence: liningxiu@163.com; pxiongfei@gmail.com

${ }^{1}$ Department of Health and Social Behavior, West China School of Public

Health and West China Fourth Hospital, Sichuan University, Chengdu, China

${ }^{5}$ The George Institute for Global Health, Faculty of Medicine, University of New South Wales, Sydney, Australia

Full list of author information is available at the end of the article

\section{Background}

Metabolic syndrome (MetS) is a pathological state of metabolic disorder that comprises elevated blood pressure (BP), dysglycemia, dyslipidemia, and abdominal obesity [1]. Although the criteria for MetS vary slightly between countries, one out of four is estimated to have MetS globally [2]. With high prevalence, MetS contributes to $30-52 \%$ of diabetes, $12-17 \%$ of cardiovascular disease, and $6-7 \%$ of all-cause mortality [3]. In the 
context of rapid lifestyle transitions, MetS is emerging as one of the leading public health challenges worldwide.

An excessive level of serum uric acid (SUA) can stimulate oxidative stress and inflammation, reduce the nitric oxide bioavailability, and accelerate the development of insulin resistance, which suggests a possible role of elevated SUA in the impairment of metabolic homeostasis $[4,5]$. Since MetS is a frequent comorbidity among patients with hyperuricemia, there has been a hypothesis for positive associations between higher SUA levels and incident MetS. This hypothesis was confirmed in a meta-analysis of cohort studies from several countries six years ago [6]. However, most of the included cohort studies were conducted in non-Chinese populations, or only assessed the relationship of one-time baseline SUA level and incident MetS. The only included Chinese study was based on secondary analyses of health check-up data from a single health examination center [7], and large population-based cohort studies on this topic were still sparse in Chinese settings. In addition, two recent studies in other populations that focused on the longitudinal changes in SUA showed inconsistent findings $[8,9]$. Since the national prevalence of MetS could reach up to $33.9 \%$ among adults in China [10], we intended to revisit the issue using national population-based longitudinal data that contain repeated measures of SUA.

Capitalizing on a nation-wide cohort study in China, we assessed the associations of baseline SUA and SUA changes with risk of MetS among middle-aged and elderly adults, and examined whether the associations differed across subpopulations. As multiple new cohort studies were published across the globe since the last meta-analysis, we also conducted a comprehensive metaanalysis of all cohort studies to update the evidence on this topic.

\section{Methods}

\section{Study design and population}

The China Health and Retirement Longitudinal Study (CHARLS) is an ongoing national population-based cohort study that is conducted to address aging-related research questions among middle-aged and elderly adults ( $\geq 45$ years) in China. The study design of CHARLS was detailed elsewhere [11]. Using a four-stage, stratified, cluster probability sampling design, 17,708 participants were enrolled from 450 rural villages or urban communities of 28 provinces in China at baseline (2011-2012). Data were collected by questionnaires, physical measurements, and biomarker measurements at baseline and during follow-up until 2015-2016. The cohort study was approved by the Biomedical Ethics Review Committee of Peking University (IRB00001052-11015), and all participants provided informed consent.
Of 17,708 study participants, 10,131 completed questionnaires and provided blood samples at baseline. We excluded the participants due to lack of information on SUA $(n=163)$ or MetS $(n=456)$ at baseline, or presence of MetS at baseline $(n=2,551)$. Of the eligible participants, we excluded participants who did not participate in the follow-up survey in 2015-2016 $(n=747)$, or who lacked information on MetS during follow-up in 2015$2016(n=2,435)$. Finally, 3,779 participants were included in our analyses (Additional file 1: Figure S1). The 3182 participants who were excluded were more likely to be older, from urban areas, have lower BMI, and higher SUA levels, but were less likely to be educated, married, and from rural areas compared to the included 3779 participants $(P \leq 0.003$ for all, Additional file 1: Table S1).

\section{Data collection}

Structured questionnaires were used at baseline to collect information including demographics, socioeconomic status, health-related behaviors, and medical history by trained interviewers [11]. Age was confirmed at baseline (2011-2012). Residence was classified into rural and urban areas. Education level was categorized into illiterate, primary school, and middle school or above. Marital status was categorized into married and unmarried (including separated, divorced, widowed, and never married). Smoking and drinking were grouped as never, former, and current smoking/drinking. Current smoking was defined as having the habit of smoking cigarettes, cigars, tobacco, or pipe currently. Current drinking was defined as having drunk any alcoholic beverages (wine, beer, or liquor) for more than once a month in the past year.

Physical measurements were collected at baseline and the follow-up in 2015-2016. BP was measured three times at 45-s intervals with an electronic sphygmomanometer on the left arm, and an average of three readings was used for analyses. Waist circumference (WC) was horizontally measured at the level of the navel using a soft measure tape. Body weight and height were measured using a digital weight scale and stadiometer. Body mass index (BMI) was calculated as the body weight in kilograms divided by the square of the height in meters $\left(\mathrm{kg} / \mathrm{m}^{2}\right)$.

Biomarker measures were completed at baseline and in 2015-2016. SUA was measured by the UA plus method. Fasting blood glucose (FBG), triglyceride (TG), and highdensity lipoprotein cholesterol (HDL-C) were measured by the enzymatic colorimetric test. Glycosylated hemoglobin (HbA1c) was measured by high performance liquid chromatography. Prevalent diabetes at baseline was defined as $F B G \geq 126 \mathrm{mg} / \mathrm{dL}, \mathrm{HbA} 1 \mathrm{c} \geq 6.5 \%$, or self-reported doctor diagnosis [12]. Rate-blanked and 
compensated Jaffe creatinine method was used to measure serum creatinine. eGFR were calculated using the Chronic Kidney Disease Epidemiology Collaboration Study equation: systolic BP (mmHg), FBG (mg/dL), TG (mg/dL), HDL-C $(\mathrm{mg} / \mathrm{dL})$, and eGFR $\left(\mathrm{mL} / \mathrm{min} / 1.73 \mathrm{~m}^{2}\right.$, all as continuous variables) were additionally adjusted for in Model 2. Similar analyses were done when the absolute and per-

$$
\mathrm{eGFR}=141 \times \operatorname{minS} c r / \kappa, 1^{\alpha} \times \max (\operatorname{Scr} / \kappa, 1)^{-1.209} \times 0.993^{\mathrm{Age}} \times 1.108[\text { if female }] \times 1.159 \text { [if black], }
$$

where Scr is serum creatinine, $\kappa$ is 0.7 for females and 0.9 for males, $\alpha$ is -0.329 for females and -0.411 for males, min means the minimum of Scr $/ \kappa$ or 1 , and max means the maximum of $\operatorname{Scr} / \kappa$ or 1 [13].

\section{Definitions of exposure and outcome}

The primary exposure was SUA levels at baseline, which was assessed as sex-specific quartiles. In additional analyses, hyperuricemia was defined as a level of $\mathrm{SUA} \geq 7.0 \mathrm{mg} / \mathrm{dL}$ in men or $\geq 6.0 \mathrm{mg} / \mathrm{dL}$ in women [14]. The absolute and percentage changes in SUA over 4 years, assessed as the difference between the SUA levels at baseline and during follow-up in 2015-2016, were the secondary exposure.

The outcome was incident MetS that was diagnosed in the last follow-up among participants who did not have MetS at baseline. MetS was defined according to the criteria from the Chinese guidelines for the management of dyslipidemia in adults [15]. MetS was diagnosed if at least three out of five criteria were met: (1) abdominal obesity (WC $\geq 90 \mathrm{~cm}$ in men or $\geq 85 \mathrm{~cm}$ in women); (2) elevated FBG ( $\geq 110 \mathrm{mg} / \mathrm{dL})$ or drug treatment of elevated glucose; (3) elevated BP $(\geq 130 / 85 \mathrm{mmHg})$ or antihypertensive drug treatment in people with a history of hypertension; (4) elevated TG ( $\geq 150 \mathrm{mg} / \mathrm{dL}$ ); and (5) reduced HDL-C $(<40 \mathrm{mg} / \mathrm{dL})$.

\section{Statistical analyses}

Data were summarized as mean and standard deviation (SD) for continuous variables, and as frequency and percentage for categorical variables. Differences of basic characteristics were compared using ANOVA (for continuous variables) and chi-square test (for categorical variables) across sex-specific SUA groups.

Logistic regression was used to estimate odds ratios (ORs) and corresponding 95\% confidence intervals (CIs) for associations of baseline SUA with incident MetS. Potential confounders were adjusted for in regression models in a stepwise manner. In Model 1, we adjusted for baseline age (continuous, years), gender (men and women), residence (urban and rural), education level (illiterate, primary school, and middle school or above), marital status (married and single), cigarette smoking (never, former, and current), and alcohol drinking (never, former, and current). Baseline WC $(\mathrm{cm})$, BMI $\left(\mathrm{kg} / \mathrm{m}^{2}\right)$, centage changes of SUA during follow-up were assessed as quartiles, and baseline SUA was additionally adjusted for in Model 2. Linear trends across SUA quartile groups were estimated by modeling SUA as the median of each quartile. We conducted subgroup analyses by sex (men and women), age ( $45-59$ and $\geq 60$ years), and BMI $(<24$ and $\geq 24 \mathrm{~kg} / \mathrm{m}^{2}$ based on the Chinese cut-offs for overweight and obesity) [16]. A product term of each stratifying variable and SUA groups was added in the main logistic regression model to examine the potential effect modification (interaction) using the Wald test. We conducted a sensitivity analysis to account for confounding from comorbidities by excluding the participants with prevalent hypertension or diabetes at baseline. We also conducted analyses of the association of combined baseline SUA and absolute SUA changes with incident MetS by generating four categories based on their median values (low baseline/minimal increase, high baseline/ minimal increase, low baseline/high increase, and high baseline/high increase). In addition, we assessed the associations of baseline SUA and absolute SUA changes with MetS components. SPSS 20.0 (IBM Co., Armonk, NY, USA) was used for all statistical analyses. Twotailed $P$ values less than 0.05 were considered statistically significant.

\section{Meta-analysis}

We conducted a meta-analysis of effect estimates from our study and other cohort studies that examined the relationship between the baseline SUA levels and MetS risk in adults. PubMed, ISI Web of Science, and Embase were searched for cohort studies up to January 30, 2021, using a search strategy that combined $\mathrm{MeSH}$ terms and keywords for SUA, hyperuricemia, or gout, metabolic syndrome, and cohort study. Eligibility criteria for metaanalyses included: (1) original cohort studies; (2) assessing the association between baseline SUA and incident MetS; (3) reporting effect estimates (either HR or OR) and $95 \% \mathrm{CI}$; (4) conducted in adults; and (5) a minimum follow-up duration of 1 year. The quality of included studies was assessed using the Newcastle-Ottawa quality assessment scale [17].

Basic characteristics and fully adjusted effect estimates were extracted from each individual study. Literature search and data extraction were performed by 
two investigators (S.C. and N.W.), and any discrepancies were resolved by consensus. Heterogeneity across studies was evaluated using the $\mathrm{Q}$ test. Random-effects (when $\mathrm{Q}$ test's $P<0.01$ ) or fixed-effects models (when $\mathrm{Q}$ test's $P>0.01)$ were applied to obtain an overall relative risk (RR) for the highest SUA level category compared with the lowest SUA level category and each $1 \mathrm{mg} / \mathrm{dL}$ increase in the SUA level. When studies separately reported effect estimates for men and women or different age subgroups, effect estimates were pooled in individual studies by using random effect meta-analysis first, and pooled estimates were combined with those from other studies for the final meta-analysis. If effect estimates were presented as ORs in identified studies, they were converted to RRs using the formula $(R R=O R /([1-p R e f]+[p R e f \times O R])$, where $p$ Ref is the prevalence of the outcome in the reference group [18]. HRs were considered as approximate RRs for the meta-analysis. Subgroup analyses were done by region (Asian, America and Europe), sex (men and women), mean age $(<45,45-65$, and $\geq 66$ years), and median follow-up time ( $<4$ and $\geq 4$ years), and subgroup heterogeneity was assessed by the meta-regression. Publication bias in the meta-analysis was assessed by the funnel plot, Egger's test, and Begg's test. Stata 16.0 (Stata Corp LLC) was used for the meta-analysis.

\section{Results}

\section{Baseline characteristics}

Of the included 3,779 participants, the mean (SD) age and BMI were $59.5(8.7)$ years and $22.7(3.3) \mathrm{kg} / \mathrm{m}^{2}$. $47.2 \%$ were men and $70.1 \%$ were rural residents. Overall, the average SUA level was significantly higher in men than in women $(5.0[1.3]$ vs. $4.0[1.1] \mathrm{mg} / \mathrm{dL}, P<0.001)$. Participants in the fourth baseline SUA quartile group were more likely to be older, urban residents, and alcohol drinkers, and to have higher BMI, WC, BP, TG, and serum creatine compared with those in the lowest quartile group (Table 1).

\section{Association between SUA levels and incident MetS}

A total of 452 participants developed MetS during a follow-up of 4 years. In the fully adjusted model, the higher quartiles of baseline SUA were significantly associated with increased incident MetS. Compared to the lowest quartile of baseline SUA, the multivariable-adjusted OR $(95 \% \mathrm{CI})$ for MetS in other three quartiles were 1.08 (0.77-1.50), 1.32 (0.95-1.82), and 1.55 (1.12-2.16) (Table 2). There was a linear trend in the odds of MetS across quartiles of baseline SUA ( $P$ for trend $=0.004$ ). Each increment of $1 \mathrm{mg} / \mathrm{dL}$ in baseline SUA was

Table 1 Baseline characteristics across quartiles of baseline SUA in the CHARLS

\begin{tabular}{|c|c|c|c|c|c|}
\hline \multirow[t]{2}{*}{ Baseline characteristics } & \multicolumn{4}{|c|}{ Quartiles of baseline SUA ${ }^{\dagger}$} & \multirow[t]{2}{*}{$P$ value* } \\
\hline & Quartile $1(N=941)$ & Quartile $2(N=948)$ & Quartile $3(N=944)$ & Quartile $4(N=946)$ & \\
\hline Men, $n(\%)$ & $445(47.3)$ & $446(47.0)$ & $446(47.2)$ & $446(47.1)$ & 0.999 \\
\hline Age, mean (SD), years & $58.7(8.5)$ & $59.1(8.5)$ & $59.6(8.7)$ & $60.8(9.0)$ & $<0.001$ \\
\hline BMI, mean (SD), kg/m² & $22.4(3.3)$ & $22.5(3.4)$ & $22.7(3.0)$ & $23.1(3.4)$ & $<0.001$ \\
\hline Urban residence, $n(\%)$ & $232(24.7)$ & $254(26.8)$ & $320(33.9)$ & $324(34.2)$ & $<0.001$ \\
\hline Illiterate, n (\%) & $269(28.6)$ & $267(28.2)$ & $245(26.0)$ & $263(27.8)$ & 0.665 \\
\hline Married, $n(\%)$ & $837(88.9)$ & $851(89.8)$ & $851(90.1)$ & $829(87.6)$ & 0.304 \\
\hline Current smokers, $n(\%)$ & $316(33.6)$ & $298(31.4)$ & $284(30.1)$ & $297(31.4)$ & 0.640 \\
\hline Current alcohol drinkers, $n$ (\%) & $218(23.2)$ & $224(23.6)$ & $260(27.5)$ & $268(28.3)$ & 0.001 \\
\hline WC, mean (SD), cm & $81.6(8.4)$ & $82.3(8.7)$ & $82.8(8.5)$ & $84.0(9.4)$ & $<0.001$ \\
\hline Systolic BP, mean (SD), mmHg & $124.2(19.1)$ & $125.2(20.1)$ & $126.5(20.5)$ & $129.3(20.5)$ & $<0.001$ \\
\hline Diastolic BP, mean (SD), mmHg & $73.2(11.4)$ & $73.0(11.6)$ & $73.9(12.0)$ & $75.0(11.7)$ & 0.001 \\
\hline $\mathrm{FBG}$, mean $(\mathrm{SD}), \mathrm{mg} / \mathrm{dL}^{\ddagger}$ & $105.0(33.6)$ & $102.6(21.5)$ & $102.7(19.6)$ & $103.1(21.0)$ & 0.105 \\
\hline $\mathrm{TG}$, mean $(\mathrm{SD}), \mathrm{mg} / \mathrm{dL}^{\ddagger}$ & $95.5(44.1)$ & $97.0(44.5)$ & $103.9(58.2)$ & $113.1(59.5)$ & $<0.001$ \\
\hline $\mathrm{HDL}-\mathrm{C}$, mean $(\mathrm{SD}), \mathrm{mg} / \mathrm{dL}^{\ddagger}$ & $54.3(14.0)$ & $55.3(14.1)$ & $55.1(14.4)$ & $54.5(14.1)$ & 0.358 \\
\hline Serum creatine, mean (SD), mg/dL & $0.69(0.15)$ & $0.74(0.15)$ & $0.77(0.16)$ & $0.85(0.22)$ & $<0.001$ \\
\hline eGFR, mean (SD), mL/min/1.73 $\mathrm{m}^{2}$ & $99.4(10.6)$ & $95.1(11.6)$ & $92.8(11.7)$ & $85.9(15.2)$ & $<0.001$ \\
\hline
\end{tabular}

BMI, body mass index; BP, blood pressure; CHARLS, China Health and Retirement Longitudinal Study; eGFR, estimated glomerular filtration rate; FBG, fasting blood glucose; HDL-C, high density lipoprotein cholesterol; SUA, serum uric acid; TG, triglycerides; WC, waist circumference

${ }^{\dagger}$ Cut-off values of baseline SUA quartiles in men: $<3.9 \mathrm{mg} / \mathrm{dL}, 3.9$ to $<4.7 \mathrm{mg} / \mathrm{dL}, 4.7$ to $<5.2 \mathrm{mg} / \mathrm{dL}, \geq 5.2 \mathrm{mg} / \mathrm{dL}$. Cut-off values of baseline SUA quartiles in women: $<3.2 \mathrm{mg} / \mathrm{dL}, 3.2$ to $<3.7 \mathrm{mg} / \mathrm{dL}, 3.7$ to $<4.3 \mathrm{mg} / \mathrm{dL}$, $\geq 4.3 \mathrm{mg} / \mathrm{dL}$

*ANOVA (for continuous variables) and chi-square test (for categorical variables) were used to compare basic characteristics across SUA quartile groups

${ }^{\ddagger}$ Conversion factors for units: $\mathrm{FBG}$ in $\mathrm{mg} / \mathrm{dL}$ to $\mathrm{mmol} / \mathrm{L}, \times 0.0555 ; \mathrm{TG}$ in $\mathrm{mg} / \mathrm{dL}$ to $\mathrm{mmol} / \mathrm{L}, \times 0.0113 ; \mathrm{HDL}-\mathrm{C}$ in $\mathrm{mg} / \mathrm{dL}$ to $\mathrm{mmol} / \mathrm{L}, \times 0.0259$ 
Table 2 ORs and 95\% Cls for incident MetS according to SUA levels in the CHARLS

\begin{tabular}{|c|c|c|c|c|c|}
\hline & MetS/total (\%) & Model 1 & Model 2 & Model 3 & $P$ for trend* \\
\hline Quartiles of baseline SUA & & & & & 0.004 \\
\hline Quartile 1 & 84/941 (8.9) & Reference & Reference & Reference & \\
\hline Quartile 2 & 93/948 (9.8) & $1.11(0.81-1.51)$ & $1.10(0.81-1.50)$ & $1.08(0.77-1.50)$ & \\
\hline Quartile 3 & $117 / 944(12.4)$ & $1.44(1.07-1.94)$ & $1.40(1.04-1.88)$ & $1.32(0.95-1.82)$ & \\
\hline Quartile 4 & $158 / 946(16.7)$ & $2.05(1.54-2.71)$ & $1.99(1.50-2.65)$ & $1.55(1.12-2.16)$ & \\
\hline Per $1 \mathrm{mg} / \mathrm{dL}$ increase & - & $1.20(1.11-1.29)$ & $1.30(1.19-1.42)$ & $1.19(1.07-1.33)$ & \\
\hline \multicolumn{6}{|l|}{ Baseline hyperuricemia } \\
\hline No & $423 / 3,645(11.6)$ & Reference & Reference & Reference & \\
\hline Yes & 29/134 (21.6) & $2.10(1.38-3.21)$ & $2.14(1.39-3.29)$ & $1.47(0.89-2.38)$ & \\
\hline $\begin{array}{l}\text { Quartiles of absolute changes in } \\
\text { SUA during follow-up }\end{array}$ & & & & & $<0.001$ \\
\hline Quartile 1 & 93/945 (9.8) & Reference & Reference & Reference & \\
\hline Quartile 2 & 101/944 (10.7) & $1.10(0.82-1.48)$ & $1.05(0.78-1.42)$ & $1.23(0.89-1.71)$ & \\
\hline Quartile 3 & $107 / 945(11.3)$ & $1.17(0.87-1.57)$ & $1.13(0.84-1.52)$ & $1.39(1.00-1.93)$ & \\
\hline Quartile 4 & $151 / 945(16.0)$ & $1.74(1.32-2.30)$ & $1.77(1.34-2.34)$ & $1.89(1.38-2.58)$ & \\
\hline $\begin{array}{l}\text { Quartiles of percent changes in } \\
\text { SUA during follow-up }\end{array}$ & & & & & $<0.001$ \\
\hline Quartile 1 & 88/944 (9.3) & Reference & Reference & Reference & \\
\hline Quartile 2 & 106/945 (11.2) & $1.23(0.91-1.66)$ & $1.19(0.88-1.61)$ & $1.32(0.95-1.82)$ & \\
\hline Quartile 3 & 113/946 (11.9) & $1.32(0.98-1.77)$ & $1.29(0.96-1.73)$ & $1.51(1.09-2.09)$ & \\
\hline Quartile 4 & $145 / 944(15.4)$ & $1.77(1.33-2.34)$ & $1.76(1.32-2.33)$ & $2.18(1.57-3.02)$ & \\
\hline
\end{tabular}

BMI, body mass index; BP, blood pressure; CHARLS, China Health and Retirement Longitudinal Study; Cl, confidence interval; eGFR, estimated glomerular filtration rate; FBG, fasting blood glucose; HDL-C, high-density lipoprotein cholesterol; Mets, metabolic syndrome; OR, odd ratio; SUA, serum uric acid; TG, triglycerides; WC, waist circumference

Model 1: non-adjusted;

Model 2: adjusted for baseline age (continuous, years), gender (men and women), residence (urban and rural), education level (illiterate, primary school, middle school or above), marital status (married and unmarried), cigarette smoking (never, former, and current), alcohol drinking (never, former, and current);

Model 3: adjusted for baseline WC (cm), BMI ( $\left.\mathrm{kg} / \mathrm{m}^{2}\right)$, systolic BP $(\mathrm{mmHg}), \mathrm{FBG}(\mathrm{mg} / \mathrm{dL}), \mathrm{TG}(\mathrm{mg} / \mathrm{dL}), \mathrm{HDL}-\mathrm{C}(\mathrm{mg} / \mathrm{dL})$, and eGFR $\left(\mathrm{mL} / \mathrm{min} / 1.73 \mathrm{~m}^{2}\right.$, all as continuous variables) in addition to covariates in Model 2. Baseline SUA (continuous, $\mathrm{mg} / \mathrm{dL}$ ) was additionally adjusted for in the analysis of quartiles of absolute changes (or percent changes) in SUA during follow-up

* $P$ values for trend were estimated by modelling the serum uric acid using the median for each quartile

${ }^{\dagger}$ Cut-off values of quartiles of absolute changes in SUA during follow-up: $<-0.1 \mathrm{mg} / \mathrm{dL},-0.1$ to $<0.5 \mathrm{mg} / \mathrm{dL}, 0.5$ to $<1.0 \mathrm{mg} / \mathrm{dL}, \geq 1.0 \mathrm{mg} / \mathrm{dL}$

${ }^{\ddagger}$ Cut-off values of quartiles of percent changes in SUA during follow-up: $<-2.4 \%,-2.4 \%$ to $<11.2 \%, 11.2 \%$ to $<26.8 \%, \geq 26.8 \%$

associated with $19 \%$ higher odds of MetS (multivariable-adjusted OR 1.19; 95\% CI 1.07-1.33). Participants with hyperuricemia had $47 \%$ higher odds of incident MetS than those without hyperuricemia (multivariableadjusted OR 1.47; 95\% CI 0.89-2.38), despite no statistical significance.

For three higher quartiles versus the lowest quartile of SUA changes (i.e., highest SUA decrease, $<-0.1 \mathrm{mg} /$ $\mathrm{dL}$ or $<-2.4 \%$ change) over 4 years, the multivariableadjusted OR (95\% CI) for MetS were 1.23 (0.89-1.71), 1.39 (1.00-1.93), and 1.89 (1.38-2.58), respectively, when the absolute change was assessed as quartiles, and 1.32 (0.95-1.82), 1.51 (1.09-2.09), and 2.18 (1.57-3.02) respectively, when the percentage change was assessed as quartiles (Table 2). Linear trends were noted in these two sets of analyses $(P$ for trend $<0.001)$.

There was no evidence of interactions of baseline SUA or absolute SUA changes with sex, age, or BMI regarding the associations with incident MetS ( $P$ for interaction $\geq 0.432$ for all, Table 3 ). There were no apparent changes in the magnitude of associations when participants with hypertension or diabetes were excluded at baseline, compared to the main analyses (Additional file 1: Table S2).

Compare to the low baseline/minimal increase category for SUA, the multivariable-adjusted OR (95\% CI) for MetS were 1.48 (1.04-2.11), 1.58 (1.13-2.21), and 1.87 (1.31-2.69), respective, for the high baseline/ minimal increase, low baseline/high increase, and high baseline/high increase (Fig. 1). There was a linear trend in the odds of MetS across the 4 categories $(P=0.008)$.

Baseline SUA were significantly associated with MetS components of elevated WC (multivariable OR [95\% CI]: 1.41 [1.03-1.89] for Quartile 3 vs. 1), FBG (1.58 [1.04-2.41] for Quartile 4 vs. 1), and TG (1.72 [1.312.26] for Quartile 4 vs. 1), while absolute SUA changes 


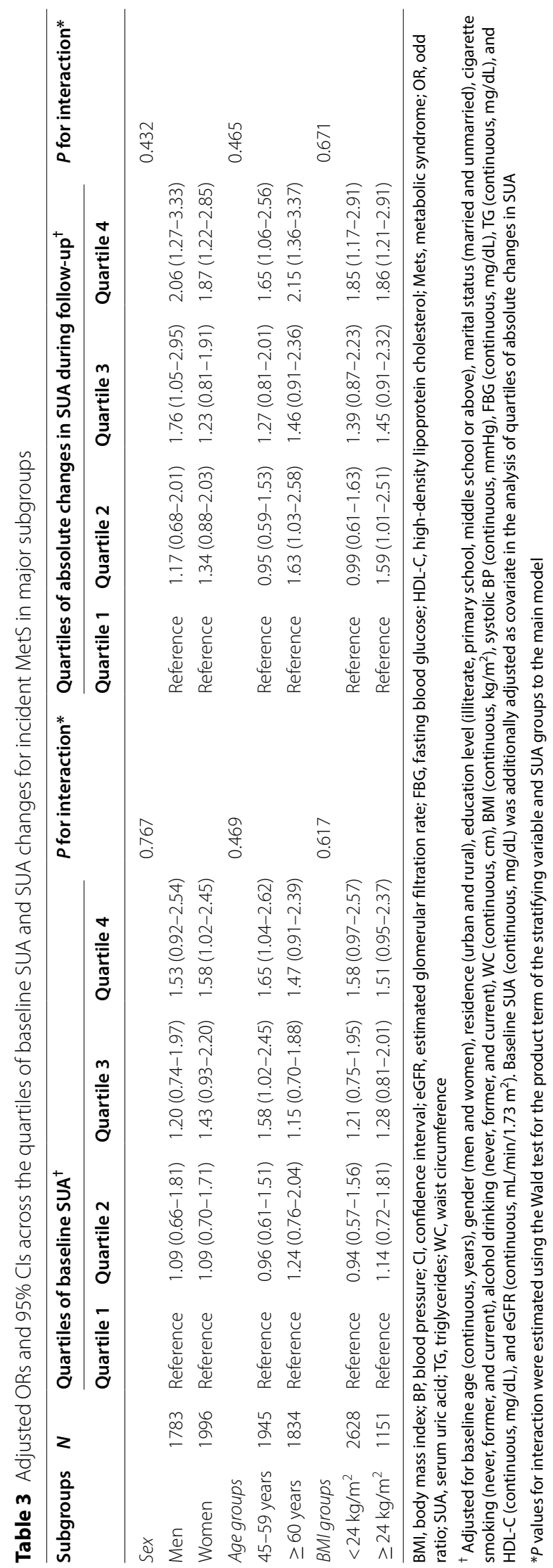




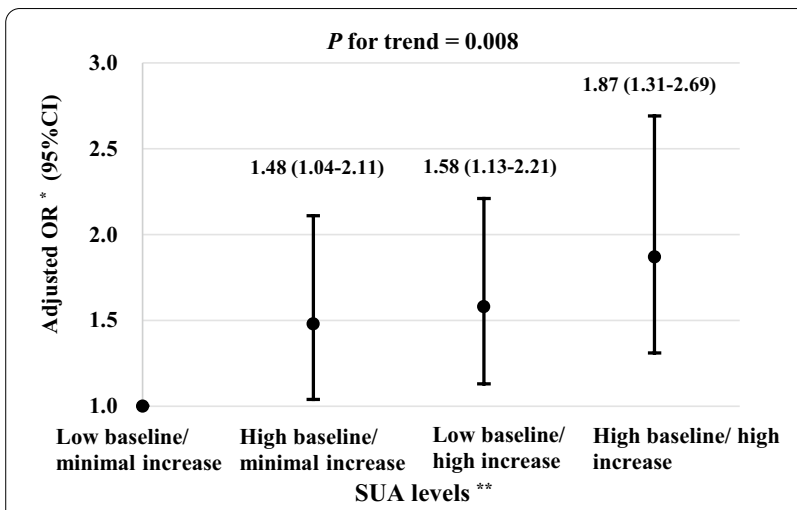

Fig. 1 Association of combined baseline SUA and SUA changes with incident metabolic syndrome in the CHARLS. Ab BMI, body mass index; $\mathrm{BP}$, blood pressure; $\mathrm{Cl}$, confidence interval; $\mathrm{CHARLS}$, China Health and Retirement Longitudinal Study; eGFR, estimated glomerular filtration rate; FBG, fasting blood glucose; HDL-C, high-density lipoprotein cholesterol; OR, odd ratio; SUA, serum uric acid; TG, triglycerides; WC, waist circumference. *Adjusted for baseline age (continuous, years), gender (men and women), residence (urban and rural), education level (illiterate, primary school, middle school or above), marital status (married and unmarried), cigarette smoking (never, former, and current), alcohol drinking (never, former, and current), WC (continuous, $\mathrm{cm}$ ), BMI (continuous, $\mathrm{kg} / \mathrm{m}^{2}$ ), systolic BP (continuous, $\mathrm{mmHg}$ ), FBG (continuous, $\mathrm{mg} / \mathrm{dL}$ ), TG (continuous, $\mathrm{mg} / \mathrm{dL}$ ), and $\mathrm{HDL}-\mathrm{C}$ (continuous, $\mathrm{mg} / \mathrm{dL}$ ), and eGFR (continuous, $\mathrm{mL} / \mathrm{min} / 1.73 \mathrm{~m}^{2}$ ). **Baseline SUA levels and absolute SUA changes during the follow-up were dichotomized based on the median values ( $4.1 \mathrm{mg} / \mathrm{dL}$ and $0.5 \mathrm{mg} / \mathrm{dL}$, respectively)

were significantly associated with MetS components of elevated WC (1.51 [1.09-2.07] for Quartile 4 vs. 1), and TG (2.21 [1.71-2.86] for Quartile 4 vs. 1; Additional file 1: Table S3).

\section{Meta-analysis for associations between SUA levels and incident MetS}

A total of 3410 records were retrieved from three databases (679 in Embase, 831 in ISI Web of Science, and 1900 in PubMed), and 1087 duplicate records were excluded. The meta-analysis included a total of 24 cohort studies (including our study, Additional file 1: Figure S2) $[7,8,19-39]$. There were 140,913 participants and 17,575 incident cases of MetS with follow-up durations ranging from 2 to 11 years (Additional file 1: Table S4). Quality assessments of all included studies showed a Newcastle-Ottawa score of 7-8 (out of 9), which indicates overall good quality (Additional file 1: Table S5). 23 studies reported effect estimates for the highest versus lowest SUA group and 9 studies reported effect estimates for each $1 \mathrm{mg} / \mathrm{dL}$ increase in the SUA level. Pooled RR (95\% CI) of incident MetS was $1.32(1.25-1.40)$ for the highest versus lowest SUA group (Fig. 2) and 1.15 (1.09-1.21) for each $1 \mathrm{mg} / \mathrm{dL}$ increase in the SUA level (Additional file 1:
Figure S3). The pooled RR was higher in the studies with median follow-up durations $\geq 4$ years than those studies with follow-up $<4$ years (pooled RR 1.38 vs. 1.29; $P$ for heterogeneity $=0.014$; Additional file 1: Table S6). No significant heterogeneity was noted between subgroups by region of studies, sex, or mean age (Additional file 1: Table S6). The funnel plot showed asymmetric distribution, and $P$ values were $<0.001$ and 0.509 for the Egger's test and Begg's test, indicating potential publication bias (Additional file 1: Figure S4).

\section{Discussion}

In this large population-based cohort, both baseline SUA levels and increases in SUA during follow-up were positively associated with higher odds of MetS in middle-aged and elderly Chinese adults. The positive associations were consolidated by findings from a comprehensive meta-analysis of 24 cohort studies among 140,913 participants.

In our cohort study, participants in the highest SUA quartile had a 1.55-fold odds of incident MetS compared to those in the lowest quartile, and the association was linear across four quartiles. Consistently, four cohort studies in Chinese populations reported positive associations between SUA levels and incident MetS [7, 29, $36,37]$, despite slight differences in the effect estimates. In particular, a large cohort study of 4988 participants showed that SUA levels were linearly associated with MetS over 9 years of follow-up in mainland China, and could be a strong and independent predictor of MetS in both men and women [29]. However, major limitations with these studies could include but were not limited to potential selection bias and residual confounding because study participants were enrolled solely from hospital settings and thus might have multiple comorbidities whose effects were difficult to adjust for in statistical analyses. In this regard, our study has been the only one that used a population-based cohort design in communities in mainland China, and our work improved the quality and generalizability of research findings on this topic in China. Consistent with our work, a few cohort studies in Korea and Japan also reported positive associations [25, $26,28,30,32,33,35]$, of which several had a sample size over 10,000 [25, 32, 35]. Besides studies in East Asians, a study among 8429 men and 1260 women in the US found that there were 1.60- and 2.29-fold odds of MetS among participants in the upper tertile $(\geq 6.5 \mathrm{mg} / \mathrm{dL})$ versus the lower tertile $(<5.5 \mathrm{mg} / \mathrm{dL})$ in a follow-up of 6.6 years [31]. Collectively, studies in different populations lent support to our finding of a positive association between baseline SUA and incident MetS in the Chinese population.

Despite strong evidence of positive association between baseline SUA and MetS, our original analyses on the link 


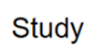

Ryu et al, 2007

Sui et al, 2008

Yang et al, 2012

Zhang et al, 2012

Goncalves et al, 2012

Zhang et al, 2013

Ferrara et al, 2014

Nagahama et al, 2014

Lee et al, 2014

Oda et al, 2014

Chen et al, 2015

Babio et al, 2015

Yadav et al, 2015

Zurlo et al, 2016

Chang et al, 2016

Yu et al, 2016

Kawamoto et al, 2018

Bombelli et al, 2018

Chen et al, 2018

Ren et al, 2018

Sumiyoshi et al, 2019

Lan et al, 2020

Chen et al, 2021

Overall (I-squared $=19.0 \%, p=0.206$ )
RR $(95 \% \mathrm{Cl}) \quad$ Weight

$1.41(0.96-2.06) 2.21$

$1.51(1.21-1.88) 6.60$

$2.46(1.31-4.62) \quad 0.80$

$1.69(1.13-2.52) 2.00$

$1.73(0.75-4.01) \quad 0.45$

$1.40(0.97-2.03) 2.33$

$1.27(1.09-1.48) \quad 13.85$

$1.47(1.12-1.93) 4.38$

$1.48(1.17-1.87) 5.78$

$1.92(0.59-6.22)$
0.23

$1.66(0.96-2.88) 1.06$

$1.40(0.94-2.09) 2.00$

$1.66(0.96-2.88) 1.06$

$1.24(0.82-1.87) \quad 1.90$

$\rightarrow 1.56(0.84-2.90) \quad 0.83$

$1.27(1.10-1.47) \quad 15.19$

1.85 (0.93-3.67) 0.68

$1.76(0.55-5.61) \quad 0.24$

$1.11(0.99-1.24) \quad 26.40$

$1.78(1.31-2.41) \quad 3.43$

$1.41(1.07-1.86) \quad 4.22$

$0.87(0.31-2.46) \quad 0.30$

$1.45(1.10-1.93) 4.06$

$1.32(1.25-1.40) \quad 100.00$

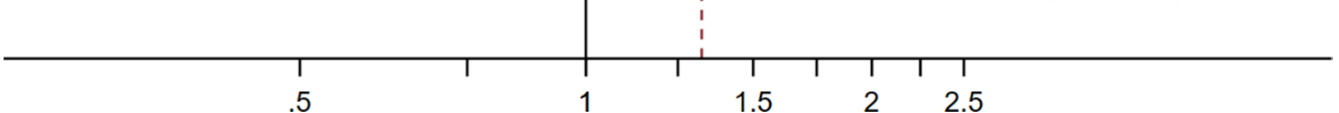

Fig. 2 RRs for the highest versus lowest level of SUA in relation to incident metabolic syndrome. Cl, confidence interval; RR, relative risk

between SUA changes and incident MetS during followup were the first done among the Chinese population. Consistent with the finding for baseline SUA, longitudinal changes of SUA over 4 years were positively associated with odds of MetS, independent of baseline SUA. Similarly, a cohort study among 407 Japanese women reported that longitudinal increase across tertile of SUA levels over 11 years was associated with higher odds of MetS during [8], while another cohort study in 6083 Norwegian adults showed that each longitudinal increase of $59 \mu \mathrm{mol} / \mathrm{L}$ in SUA over 7 years was associated with $28 \%$ increased risk of incident MetS [27]. On the contrary, another cohort study among 13,057 Korean adults showed that percentage change of SUA was inversely associated with incident MetS during the 7-year followup [9]. Of note, it selectively enrolled participants from a medical center versus communities in other three studies, which might partially explain the discrepancies in research findings due to potential different profiles of participants.

In our meta-analysis of 24 cohort studies including 140,913 participants and 17,575 cases, a $32 \%$ increase in the risk of MetS was noted in the highest versus the lowest SUA group, which was slightly lower than the effect estimate reported in the meta-analysis in 2015 (pooled RR: 1.72) [6]. Compared to these previous analyses, our current meta-analysis comprised more than twice the original studies and study participants, which substantially improves the precision of effect estimates and statistical power for subgroup analyses. We found that the association between SUA and MetS was stronger in studies with longer follow-up durations, suggesting that high SUA predicts long-term risk of MetS. As most of the included studies in our meta-analysis and our current cohort study had a short average follow-up (for example, only four had a follow-up over 6 years) [8, 20, 27, 29], reverse causality could hardly be ruled out, i.e., the association might be explained as metabolic syndrome leading to high SUA. Thus, future large-scale populationbased studies with long-term follow-ups are still warranted to dissect the effect of SUA on MetS. In addition, we observed consistent higher risk of MetS among participants with higher SUA across sex and age groups. Of note, accumulated evidence shows that elevated SUA 
levels might contribute to other cardiometabolic diseases such as diabetes, chronic kidney disease, and nonalcoholic fatty liver disease [24, 40], which supports the role of SUA in the development of MetS. Collectively, our original analyses in Chinese population and comprehensive meta-analysis across populations imply that monitoring the dynamic changes in SUA could be important for screening for high-risk individuals for MetS, especially for the middle-aged and elderly adults. From a clinical point of view, high SUA levels even below the limit for defining hyperuricemia and an increase in SUA over time might be indicative of potential higher risk of cardiometabolic diseases.

Although the underlying mechanisms for increased risk of MetS associated with higher SUA levels are not fully understood, there are several potential lines of evidence. First, elevated SUA levels have been reported to induce endothelial dysfunction and suppress endothelial nitric oxide bioavailability, leading to insulin resistance and oxidative stress that contribute to MetS [5, 41, 42]. Second, the rise in SUA is a strong predictor for the development of non-alcoholic fatty liver, which increases triglyceride accumulation as a component of MetS [43-45]. Third, experimental studies suggested that SUA may cause cardiovascular disorders such as hypertension by stimulating proliferation, angiotensin II production, and oxidative stress in vascular smooth muscle cell through renal and intracellular renin angiotensin system [46]. Fourth, diets that contain high fat and high fructose contribute to both SUA and features of metabolic syndrome [5], which implies that the association between SUA and metabolic syndrome may be partially explained by the confounding effect of unhealthy diets.

To the best of our knowledge, we conducted the first nation-wide population-based cohort study on this topic in China. It has strengths such as population-based design, large sample size, prospective design, structured questionnaire surveys for basic information, and standardized anthropometric and biochemical measurements. In addition, we updated the evidence through a comprehensive meta-analysis. However, some limitations should still be acknowledged. First, a large number of participants were lost to follow-up or did not provide sufficient information for our original analyses, and certain baseline characteristics such as age and BMI were different between included and exclude participants, which might lead to potential selection and information bias in our original work. However, our findings in the Chinese participants were corroborated by findings in the metaanalysis across populations. Second, the CHARLS did not collect detailed dietary or physical activity information so we could not adjust for the confounding effects of dietary factors such as intake of purine-rich foods, or physical activity. Third, due to data unavailability in the CHARLS, we could not exclude the participants who might use drugs known to influence SUA levels such as allopurinol, which may confound our estimates. Fourth, the current study among Chinese had a short followup so it may not capture sufficient cases for statistically powered subgroup analyses or rule out potential reverse causality.

\section{Conclusions}

Higher baseline SUA and an increase in SUA over time were associated with the development of MetS among Chinese middle-aged and elderly adults. Such positive associations were supported by findings from an additional meta-analysis across multiple populations. Although future studies are still needed to explore the underlying mechanisms, SUA levels might need to be monitored closely for future risk of MetS in clinical practice.

\section{Abbreviations}

BMI: Body mass index; BP: Blood pressure; CHARLS: China Health and Retirement Longitudinal Study; Cl: Confidence interval; eGFR: Estimated glomerular filtration rate; FBG: Fasting blood glucose; HR: Hazard ratio; HDL-C: Highdensity lipoprotein cholesterol; MetS: Metabolic syndrome; OR: Odd ratio; RR: Relative risk; SUA: Serum uric acid; TG: Triglycerides; WC: Waist circumference.

\section{Supplementary Information}

The online version contains supplementary material available at https://doi. org/10.1186/s12986-021-00584-x.

Additional file 1: Table S1. Comparison of baseline characteristics between participants included and excluded. Table S2. Association of SUA with incident MetS in participants without hypertension or diabetes at baseline. Table S3. Association of SUA with MetS components.

Table S4. Basic information of studies included in the meta-analysis. Table S5. Newcastle-Ottawa quality assessments. Table S6. Subgroup analyses for the meta-analysis. Figure $\mathbf{S 1}$. Flowchart of participant eligibility. Figure S2. Flowchart of eligibility of studies for the meta-analysis. Figure S3. Association between each $1 \mathrm{mg} / \mathrm{dL}$ increase in the SUA and incident MetS. Figure S4. Funnel plot.

\section{Acknowledgements}

We thank the China Center for Economic Research, National School of Development at Peking University for providing the CHARLS data.

\section{Authors' contributions}

Conception or design: SC, NL, and XFP. Acquisition, analysis, or interpretation of data: SC and NW. Drafting the work or revising: SC, NL, and XFP. WritingReview and Editing: $C Y, Y X, C X, Y H$, and JZ. All authors revised the manuscript critically for important intellectual content. All authors read and approved the final manuscript.

\section{Funding}

XFP was supported by the International Postdoctoral Exchange Fellowship of the China Postdoctoral Council (No. 20180062). The sponsor had no role in the study design, collection, analysis, or interpretation of data. 


\section{Availability of data and material}

The datasets used during the current study are available in the CHARLS officia website, http://charls.pku.edu.cn/. All data, analytic methods, and study materials presented within this article are available from the corresponding author on reasonable request.

\section{Declarations}

\section{Ethics approval and consent to participate}

The study was approved by the Biomedical Ethics Review Committee of Peking University (IRB00001052-11015), and informed consent was obtained from all participants.

\section{Consent for publication}

Not applicable.

\section{Competing interests}

The authors declare that they have no competing interests.

\section{Author details}

${ }^{1}$ Department of Health and Social Behavior, West China School of Public Health and West China Fourth Hospital, Sichuan University, Chengdu, China. ${ }^{2}$ Kidney Disease Center, The First Affiliated Hospital, College of Medicine, Zhejiang University, Hangzhou, China. ${ }^{3}$ Department of Gastroenterology, The First Affiliated Hospital, College of Medicine, Zhejiang University, Hangzhou, China. ${ }^{4}$ Department of Cardiology, Shunde Hospital, Southern Medical University, Foshan, China. ${ }^{5}$ The George Institute for Global Health, Faculty of Medicine, University of New South Wales, Sydney, Australia. ${ }^{6}$ The Ministry of Education Shanghai Key Laboratory of Children's Environmental Health, Xinhua Hospital, Shanghai Jiao Tong University School of Medicine, Shanghai, China. ${ }^{7}$ Division of Epidemiology, Department of Medicine, Vanderbilt University Medical Center, Vanderbilt Epidemiology Center, Nashville, USA. ${ }^{8}$ Department of Epidemiology and Biostatistics, and Ministry of Education Key Lab of Environment and Health, School of Public Health, Tongji Medical College, Huazhong University of Science and Technology, Wuhan, China.

Received: 9 April 2021 Accepted: 27 May 2021

Published online: 09 June 2021

\section{References}

1. Alberti KG, Eckel RH, Grundy SM, Zimmet PZ, Cleeman Jl, Donato KA, Fruchart JC, James WP, Loria CM, Smith SC. Harmonizing the metabolic syndrome. Circulation. 2009;120:1640-5.

2. Saklayen MG. The global epidemic of the metabolic syndrome. Curr Hypertens Rep. 2018;20:12.

3. Ford ES. Risks for all-cause mortality, cardiovascular disease, and diabetes associated with the metabolic syndrome: a summary of the evidence. Diabetes Care. 2005;28:1769-78.

4. Lima WG, Martins-Santos ME, Chaves VE. Uric acid as a modulator of glucose and lipid metabolism. Biochimie. 2015;116:17-23.

5. Kanbay M, Jensen T, Solak Y, Le M, Roncal-Jimenez C, Rivard C, Lanaspa MA, Nakagawa T, Johnson RJ. Uric acid in metabolic syndrome: from an innocent bystander to a central player. Eur J Intern Med. 2016;29:3-8.

6. Yuan H, Yu C, Li X, Sun L, Zhu X, Zhao C, Zhang Z, Yang Z. Serum uric acid levels and risk of metabolic syndrome: a dose-response meta-analysis of prospective Studies. J Clin Endocrinol Metab. 2015;100:4198-207.

7. Chen DN, Zhang HY, Gao Y, Lu Z, Yao ZT, Jiang YH, Lin XG, Wu CL, Yang $\mathrm{XB}$, Tan AH, et al. Cross-sectional and longitudinal associations between serum uric acid and metabolic syndrome: results from Fangchenggang Area Male Health and Examination Survey in China. Clin Chim Acta. 2015;446:226-30.

8. Kawamoto R, Ninomiya D, Kasai Y, Senzaki K, Kusunoki T, Ohtsuka N, Kumagi T. Baseline and changes in serum uric acid independently predict 11-year incidence of metabolic syndrome among community-dwelling women. J Endocrinol Invest. 2018;41:959-68.

9. Yu TY, Jin SM, Jee JH, Bae JC, Lee MK, Kim JH. The protective effects of increasing serum uric acid level on development of metabolic syndrome. Diabetes Metab J. 2019:43:504-20.
10. Lu JL, Wang LM, Li M, Xu Y, Jiang Y, Wang WQ, Li JH, Mi SQ, Zhang M, Li $Y C$, et al. Metabolic syndrome among adults in China: the 2010 China noncommunicable disease surveillance. J Clin Endocrinol Metab. 2017;102:507-15

11. Zhao Y, Hu Y, Smith JP, Strauss J, Yang G. Cohort profile: the China health and retirement longitudinal study (CHARLS). Int J Epidemiol. 2014:43:61-8.

12. Association AD. Classification and diagnosis of diabetes: standards of medical care in diabetes-2021. Diabetes Care. 2021:44:515-33.

13. Levey AS, Stevens LA, Schmid CH, Zhang YL, Castro AF 3rd, Feldman HI, Kusek JW, Eggers P, Van Lente F, Greene T, et al. A new equation to estimate glomerular filtration rate. Ann Intern Med. 2009;150:604-12.

14. Liu H, Zhang X-M, Wang Y-L, Liu B-C. Prevalence of hyperuricemia among Chinese adults: a national cross-sectional survey using multistage, stratified sampling. J Nephrol. 2014;27:653-8.

15. Joint committee issued Chinese guideline for the management of dyslipidemia in adults. 2016 Chinese guideline for the management of dyslipidemia in adults (in Chinese). Zhonghua Xin Xue Guan Bing Za Zhi. 2016; 44:833-53.

16. Pan XF, Wang L, Pan A. Epidemiology and determinants of obesity in China. Lancet Diabetes Endocrinol. 2021;9:373-92.

17. Stang A. Critical evaluation of the Newcastle-Ottawa scale for the assessment of the quality of nonrandomized studies in meta-analyses. Eur J Epidemiol. 2010;25:603-5.

18. Zhang J, Yu KF. What's the relative risk? A method of correcting the odds ratio in cohort studies of common outcomes. JAMA. 1998;280:1690-1.

19. Babio N, Martinez-Gonzalez MA, Estruch R, Waernberg J, Recondo J, Ortega-Calvo M, Serra-Majem L, Corella D, Fito M, Ros E, et al. Associations between serum uric acid concentrations and metabolic syndrome and its components in the PREDIMED study. Nutr Metab Cardiovasc Dis. 2015:25:173-80.

20. Bombelli M, Quarti-Trevano F, Tadic M, Facchetti R, Cuspidi C, Mancia G, Grassi G. Uric acid and risk of new-onset metabolic syndrome, impaired fasting glucose and diabetes mellitus in a general Italian population: data from the Pressioni Arteriose Monitorate E Loro Associazioni study. J Hypertens. 2018;36:1492-8.

21. Chang JB, Chen YL, Hung YJ, Hsieh CH, Lee CH, Pei D, Lin JD, Wu CZ, Liang $Y J$, Lin CM. The role of uric acid for predicting future metabolic syndrome and type 2 diabetes in older people. J Nutr Health Aging. 2016;21:329-35.

22. Chen Y, Kao T, Yang H, Chou C, Wu C, Lai C, Sun Y, Wang C, Chen W. The association of uric acid with the risk of metabolic syndrome, arterial hypertension or diabetes in young subjects - an observational study. Clin Chim Acta. 2018;478:68-73.

23. Goncalves JP, Oliveira A, Severo M, Santos AC, Lopes C. Cross-sectional and longitudinal associations between serum uric acid and metabolic syndrome. Endocrine. 2012;41:450-7.

24. Lan Q, Wu H, Zhou XH, Zheng L, Lin F, Meng QS, Xi XL, Yue AX, Buys $\mathrm{N}$, Sun $\mathrm{J}$, et al. Predictive value of uric acid regarding cardiometabolic disease in a community-dwelling older population in Shanghai: a cohort study. Front Med. 2020;7:24.

25. Lee JK, Ryoo JH, Choi JM, Park SK. Serum uric acid level and the incidence of metabolic syndrome in middle-aged Korean men: a 5-year follow-up study. J Prev Med Public Health. 2014;47:317-26.

26. Nagahama K, Inoue T, Kohagura K, Ishihara A, Kinjo K, Ohya Y. Hyperuricemia predicts future metabolic syndrome: a 4-year follow-up study of a large screened cohort in Okinawa. Jpn Hypertens Res. 2014;37:232-8.

27. Norvik JV, Storhaug HM, Ytrehus K, Jenssen TG, Zykova SN, Eriksen BO, Solbu MD. Overweight modifies the longitudinal association between uric acid and some components of the metabolic syndrome: the Tromso Study. BMC Cardiovasc Disord. 2016;16:85.

28. Oda E. Serum uric acid is an independent predictor of metabolic syndrome in a Japanese health screening population. Heart Vessels. 2014;29:496-503.

29. Ren P, Gao MN. Association between metabolic syndrome and the serum uric acid: a cohort Study. Clin Lab. 2018;64:719-26.

30. Ryu S, Song J, Choi BY, Lee SJ, Kim WS, Chang Y, Kim DI, Suh BS, Sung KC. Incidence and risk factors for metabolic syndrome in Korean male workers, ages 30 to 39. Ann Epidemiol. 2007;17:245-52.

31. Sui X, Church TS, Meriwether RA, Lobelo F, Blair SN. Uric acid and the development of metabolic syndrome in women and men. Metab Clin Exp. 2008;57:845-52. 
32. Sumiyoshi H, Ohyama Y, Imai K, Kurabayashi M, Saito Y, Nakamura T. Association of uric acid with incident metabolic syndrome in a Japanese general population. Int Heart J. 2019;60:830-5.

33. Yadav D, Lee ES, Kim HM, Choi E, Lee EY, Lim JS, Ahn SV, Koh SB, Chung $\mathrm{CH}$. Prospective study of serum uric acid levels and incident metabolic syndrome in a Korean rural cohort. Atherosclerosis. 2015;241:271-7.

34. Yang $\mathrm{T}$, Chu $\mathrm{CH}$, Bai CH, You SL, Chou YC, Chou WY, Chien KL, Hwang LC, Su TC, Tseng CH, et al. Uric acid level as a risk marker for metabolic syndrome: a Chinese cohort study. Atherosclerosis. 2012;220:525-31.

35. Yu TY, Jee JH, Bae JC, Jin SM, Baek JH, Lee MK, Kim JH. Serum uric acid: a strong and independent predictor of metabolic syndrome after adjusting for body composition. Metab Clin Exp. 2016;65:432-40.

36. Zhang M, Gao Y, Wang X, Chang H, Huang G. Serum uric acid and appropriate cutoff value for prediction of metabolic syndrome among Chinese adults. J Clin Biochem Nutr. 2013;52:38-42.

37. Zhang Q, Zhang C, Song X, Lin H, Zhang D, Meng W, Zhang Y, Zhu Z, Tang $F$, Liu L, et al. A longitudinal cohort based association study between uric acid level and metabolic syndrome in Chinese Han urban male population. BMC Public Health. 2012;12:419.

38. Zurlo A, Veronese N, Giantin V, Maselli M, Zambon S, Maggi S, Musacchio E, Toffanello ED, Sartori L, Perissinotto E, et al. High serum uric acid levels increase the risk of metabolic syndrome in elderly women: the PRO.V.A study. Nutr Metab Cardiovasc Dis. 2016;26:27-35.

39. Ferrara LA, Wang H, Umans JG, Franceschini N, Jolly S, Lee ET, Yeh J, Devereux RB, Howard BV, de Simone G. Serum uric acid does not predict incident metabolic syndrome in a population with high prevalence of obesity. Nutr Metab Cardiovasc Dis. 2014;24:1360-4.
40. Borghi C, Francesco A, Cicero G. Serum uric acid and cardiometabolic disease: another brick in the wall? Hypertension. 2017;69:1011-3.

41. Spatola L, Ferraro PM, Gambaro G, Badalamenti S, Dauriz M. Metabolic syndrome and uric acid nephrolithiasis: insulin resistance in focus. Metabol Clin Exp. 2018:83:225-33.

42. King C, Lanaspa MA, Jensen T, Tolan DR, Sanchez-Lozada LG, Johnson RJ. Uric acid as a cause of the metabolic syndrome. Uric Acid Chronic Kidney Dis. 2018;192:88-102.

43. Tsouli SG, Liberopoulos EN, Mikhailidis DP, Athyros VG, Elisaf MS. Elevated serum uric acid levels in metabolic syndrome: an active component or an innocent bystander? Metab Clin Exp. 2006;55:1293-301.

44. Liu Z, Que S, Zhou L, Zheng S. Dose-response relationship of serum uric acid with metabolic syndrome and non-alcoholic fatty liver disease incidence: a meta-analysis of prospective studies. Sci Rep. 2015;5:14325.

45. Choi SS, Diehl AM. Hepatic triglyceride synthesis and nonalcoholic fatty liver disease. Curr Opin Lipidol. 2008;19:295-300.

46. Corry DB, Eslami P, Yamamoto K, Nyby MD, Makino H, Tuck ML. Uric acid stimulates vascular smooth muscle cell proliferation and oxidative stress via the vascular renin-angiotensin system. J Hypertens. 2008;26:269-75.

\section{Publisher's Note}

Springer Nature remains neutral with regard to jurisdictional claims in published maps and institutional affiliations.
Ready to submit your research? Choose BMC and benefit from:

- fast, convenient online submission

- thorough peer review by experienced researchers in your field

- rapid publication on acceptance

- support for research data, including large and complex data types

- gold Open Access which fosters wider collaboration and increased citations

- maximum visibility for your research: over 100M website views per year

At BMC, research is always in progress.

Learn more biomedcentral.com/submissions 